\title{
Remote Control of Mobile Robot using the Virtual Reality
}

\author{
Ibari Benaoumeur*, Ahmed-foitih Zoubir*, and Hanifi Elhachimi Amar Reda** \\ Laboratory of Power Systems, Solar Energy and Automation L.E.P.E.S.A. University of Sciences and Technology of \\ Oran, USTO/MB. Oran, Algeria
}

\begin{tabular}{l}
\hline \hline Article Info \\
\hline Article history: \\
Received Mar 20, 2015 \\
Revised Jun 26, 2015 \\
Accepted Jul 13, 2015
\end{tabular}

\section{Keyword:}

Mobile Robot

Path Planning

Teleoperation

Virtual Reality

\begin{abstract}
In this paper we present the simulation and manipulation of teleoperation system for remote control of mobile robot using the Virtual Reality (VR). The objective of this work is to allow the operator to control and supervise a unicycle type mobile robot. In this research we followed three ways: The use of articulated robotic mobile on the Web, the design of remote environment for the experimentation using the network for the mobile robot and the architecture of control is proposed to facilitate the piloting of the robot. This work proposes a hardware and software architecture based on communication and information technologies to control the virtual robot to improve the control towards the remote robot. A path planning method is integrated to the remote control system. Results show the real possibilities offered by this manipulation, in order to follow a trajectory of the robot and to create applications with a distance access to facilities through networks like the Internet and wireless.
\end{abstract}

Copyright (C) 2015 Institute of Advanced Engineering and Science. All rights reserved.

\section{Corresponding Author:}

Ibari Benaoumeur

Laboratory of Power Systems, Solar Energy and Automation L.E.P.E.S.A

University of sciences and technology of Oran USTO/MB

El Mnaouar, BP 1505, Bir El Djir, Oran, Algeria 31000

Phone +213(0) 41560329

Email ibarib@yahoo.fr

\section{INTRODUCTION}

Nowadays, the technology of the remote control has evolved considerably because it must respond to the increasing need to the remote control various devices designed for use in specialized or general public applications. In this ways, we can design effective systems for teleoperation able to control complex robotic devices by using the new techniques of virtual reality and augmented reality.

Robotics teleoperation systems are widely used in: industry, science, medicine, education, entertainment and military applications [1]. Successful applications of remotely controled mobile robots in different environments are presented in $[2,3,4]$. The teleportation of mobile robots is classified in three categories: exploration rovers, Unmanned Ground Vehicles (UGV) and dangerous duty [5]. Exploration rovers are robots designed to remotely perform science tasks [6]. UGVs are used for tasks requiring remote navigation such as reconnaissance or surveillance [7]. Dangerous duty robots work in conditions which have extremely grave dangers $[8,9,10]$. The mostely used method for robot mobile teleoperation is the direct interface: the operator directs the robot with hand-controllers and watching video from cameras [11, 12]. A new control strategy for camera viewpoint positioning in teleoperation of mobile robots is presented in [13], the proposed method ensures that all important points for the remote control are included in thenvideo feed, this method was implemented and tested in teleoperation experiments with a mobile robot.

In contrast to direct interfaces, the virtual reality provides an external perspective which allows the operator to drive/pilot the vehicle from the outside. VR technology [14, 15] has been used in industrial companies as well, especially in telerobotic virtual design and machining simulation system because they offer lower operating costs than the real situation $[16,17,18,19]$. Virtual reality environment has long been 
adopted as an effective way to address time delay problems in robot teleoperation system [20], and can surmount the influence of time delay in signal transmission by predictive display [21].

In term of supervisory control, In [22] Cooper et al. described how earth-based operators used the Rover Control Workstation to control the Sojourner rover on Mars. Nguyen et al. [23] described several virtual reality based interfaces for exploration, this virtual reality based interface concisely displays many data sets: stereo-vision based terrain, digital elevation map, and simulated descent images.

There are many research articles emphasizing the importance of VR application at the remote control. An approach to designing a Virtual Robot for control system design is presented in [24], robotic Simulation Environment is introduced for testing and visualization of robotic algorithms, but the testing on an real robot is much longer and tedious process [25]. In the design, assembly and manufacturing area, there has been a lot of research and development and a numbers of software tools have been developed in this field. Liang et al.[26] developed an appropriate methodology with open architecture for real-time monitoring and remote control of networked mobile Robot and a new enabling technology to bring traditional robotic tools on-line with combined monitoring and control capability is presented to manipulate the wheeled mobile robot data and to carry out a variety of assembly functions. A distributed platform that allows the special group of user to control a gadget (possibly a robot) through internet as a medium is presented in [27], a fuzzy logic controller is used to control the robots motion along a predefined path with the necessary manipulation of the normal course. In [28] the position of robot by means of both force and visual feedbacks is controlled, a force feedback joystick is used to deliver collision details to operator hand and drive 2-DOF slave robot in Virtual Reality environment.

In the term following a path many methods are developed [29, 30], These approachs are are not tested in the remote control with real implementation. Following these ideas, we are developing a system based on virtual environments, intended to facilitate the teleoperetion of a unicycle mobile robot. This system has not high-cost hardware requirement. A tele-robotic system basically consists of three main parts: the remote robot, the communication network and the user interface [31]. We use a unicycle mobile for the remote robot, the TCP/IP (Transmission Control Protocol/Internet Protocol) and wireless for the communication network and the VR environment for the user interface. The objective of this work is to allow a user to teleoperate a unicycle mobile through an intuitive human-machine interface using virtual reality as tool assistance the perception of the distant site, the Internet network as a transmission support and the wireless network between the server and the robot. In this work the interface provide tools to make a task easier and mechanisms for the operator and the robot to exchange information at different levels of detail or abstraction and to verify the robot in Virtual Reality and the wireless remote control. An additional objective is to integrate a path planning method to the remote control system, the method is tested in real unicycle robot mobile. The rest of this paper is organized as follows: In section 2, software design and Architecture of the system is described with the different modules constituting our system. The hardware architecture of the tele-robotic system is presented in section 3 . The effectiveness of the proposed following a trajectory method of the robot is verified and presented in section 4 . Finally, section 4 contains some concluding remarks.

\section{SOFTWARE DESIGN AND ARCHITECTURE}

The software architecture of the proposed station is based on modules, communicating between them using the request message. To guarantee the operator safety, the robot control is achieved out remotely via network communications so the client server connection is established using data frames transmitted via instant network communications protocols. The algorithm presented in the Figure 1 is used for verify the ip (Internet Protocol) address and the port of the server client connection. 


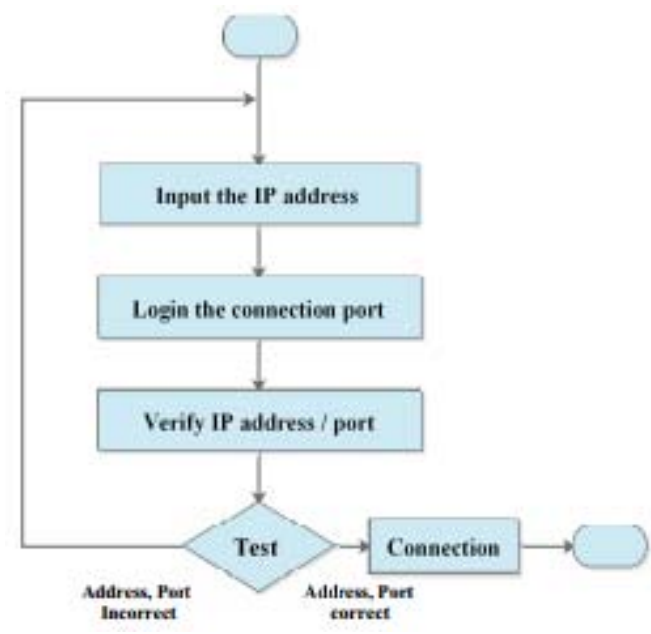

Figure 1. The Server/Client connection

\subsection{The Server Station}

The communication TCP/IP module allows the user to control the robot while letting download the graphic interface and to send orders, and the Wireless Network Protocol is used to connecting the mobile robot with the sever station as see in Figure 2, the server is targeted mainly for battery-powered applications where low data rate, low cost, and long battery life are main requirements.

To follow the evaluation of virtual robot we use a VR environment to guarantee operability and safety. The virtual Reality Modeling Language (VRML) is a file format for describing interactive 3D objects (The Virtual Robot 3d Model), having the character of being simple to use, open and interactive which make it a powerful tool for setting up the Web-based-simulating systems [32, 33, 34]. Its has a strong ability for graphics display and good Web running. To visualize to real moving of the robot with a visual feedback, a camera is added.

The Server Station is composed of principal processes as see in Figure 2 : 


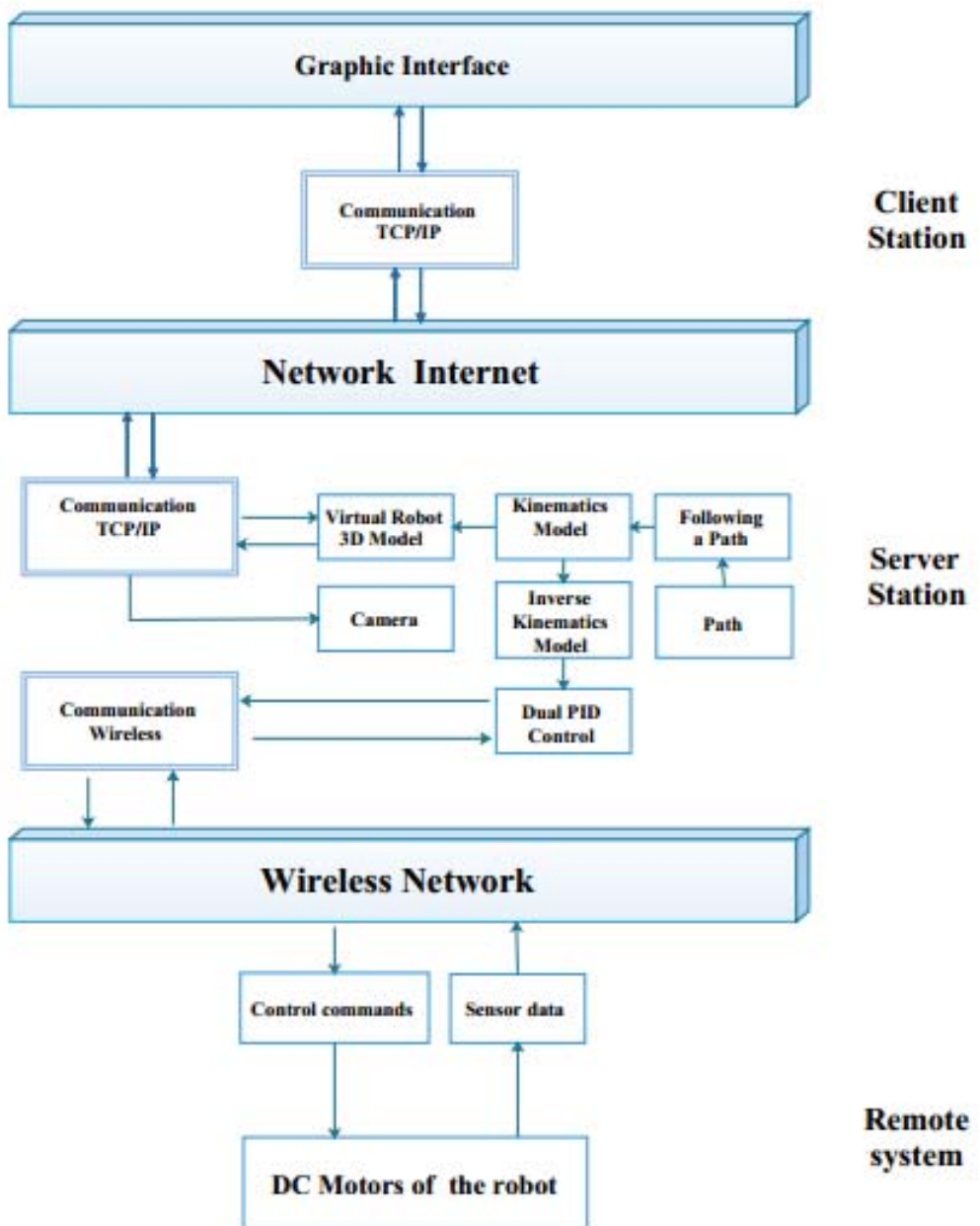

Figure 2. The software architecture of the system

\subsubsection{The Forward Kinematics of the Mobile Robot}

In this work we use a model for the unicycle type mobile robot as see in Figure 3. The configuration of the robot mobile in the workspace can be completely defined by three parameters $\mathrm{x}, \mathrm{y}$ and $\theta$. $L$ is the distance between wheels. $\omega$ is the angular velocity of the robot around the Instantaneous Center of Rotation (ICR). Respectively $v_{r}$ and $v_{l}$ are velocities of the right and left wheel, and $\omega_{r}, \omega_{l}$ are angular velocities of the right and left wheel. $r$ is wheel radius. From the Figure 3, the linear velocity $v$ is given by:

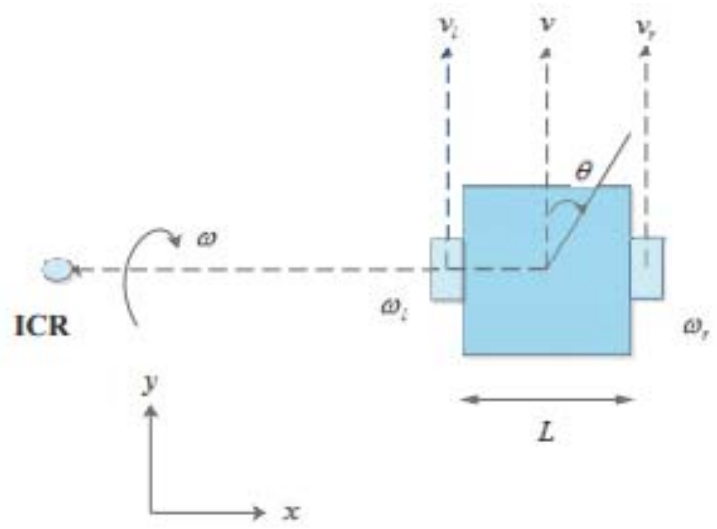

Figure 3. Parameters of the unicycle-like mobile robot 


$$
v=\frac{v_{r}+v_{l}}{2}=\frac{r\left(\omega_{r}+\omega_{l}\right)}{2}
$$

And the angular velocity of the robot is given by:

$$
\dot{\theta}=\frac{v_{r}-v_{l}}{L}=\omega=\frac{r\left(\omega_{r}-\omega_{l}\right)}{L}
$$

This kinematic model can be described by the following equations [35]:

$$
\left\{\begin{array}{c}
\dot{x}=v \cos \theta \\
\dot{y}=v \sin \theta \\
\dot{\theta}=\omega
\end{array}\right.
$$

The nonholonomic constraint that the driving wheels purely roll and do not slip must be respected, the robot cannot move in lateral direction to its wheels, which is:

$$
-\dot{x} \sin \theta+\dot{y} \cos \theta=0
$$

\subsubsection{Following a Trajectory of the Robot}

For path following, effective algorithm is proposed for trajectory tracking the goal position $x^{*}(t) \bullet y^{*}(t)$, the robot try to maintain a certain distance $d^{*}$, the error between robot position and goal position can be calculated as follows:

$$
e=\sqrt{\left(x^{2}-x\right)^{2}+\left(y^{*}-y\right)^{2}}-d^{*}
$$

we use proportional integral (PI) controller to control the robot's velocity

$$
v^{*}=k_{v}+k_{i} \int e d t
$$

and the angle $\theta^{*}$ can be expressed as follows:

$$
\theta^{*}=\tan ^{-1}\left(\frac{y^{*}-y}{x^{*}-x}\right)
$$

The path planning model of the robot is shown in Figure 4, in this model, the kinematic model of a unicycle type robot is used to turn the steering wheel. $\omega$ and $v$ are the inputs of the robot and the output $\theta$ is the current turning angle of the robot. 


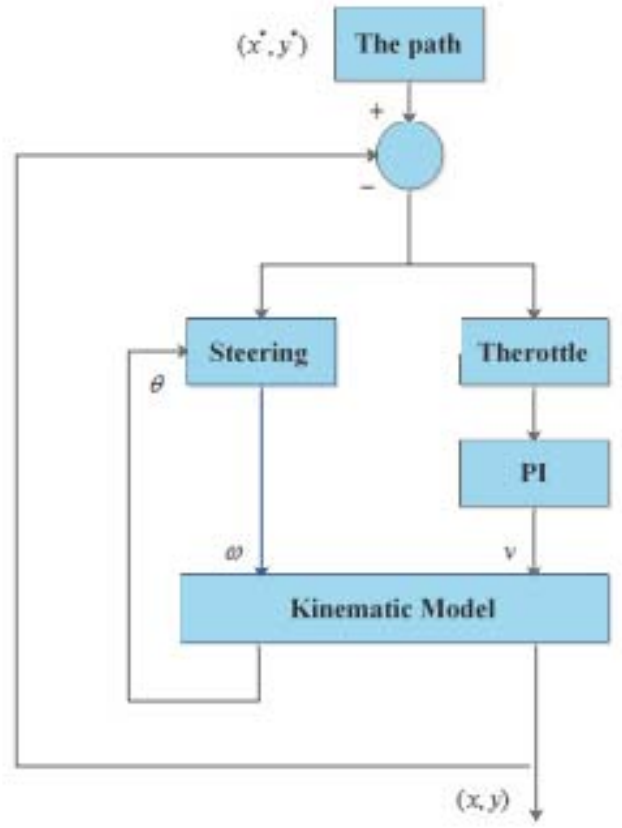

Figure 4. Following a trajectory model of the robot

\subsubsection{The Inverse Kinematics of the Robot Mobile}

The position of the center of mass in respect to turn angles of each one of the robot's wheels is determined with the direct kinematic model, the direct kinematic model of the mobile robot is expressed as:

$$
\left\{\begin{array}{c}
\dot{x}=-\frac{r \sin \theta}{2} \dot{\omega}_{l}-\frac{r \sin \theta}{2} \dot{\omega}_{r} \\
\dot{y}=\frac{r \sin \theta}{2} \dot{\omega}_{l}+\frac{r \sin \theta}{2} \dot{\omega}_{r} \\
\dot{\theta}=\frac{r}{L} \dot{\omega}_{l}-\frac{r}{2} \dot{\omega}_{r}
\end{array}\right.
$$

The inverse kinematics is to determine the angular velocity of each wheel, given the position and the orientation of the mobile robot. The inverse kinematics can be written form as follows [36].

$$
\left\{\begin{array}{l}
\dot{\omega}_{l}=-\frac{2 v-L \omega}{2 r} \\
\dot{\omega}_{r}=-\frac{2 V-L \omega}{2 r}
\end{array}\right.
$$

\subsubsection{Dual PID Control}

The PID Control block should be used to control the speed two wheels of the differentially driven vehicle robot which are driven by two separate DC motors, Finding out the values of these gains requires a certain PID controller design procedure by taking the transfer function of each motor under consideration. Several methods are available in literature for PID controller design, for example Ziegler-Nichols tuning rules, Optimization by Performance Index Reduction (ITAE), root locus and frequency-response methods $[37,38]$ etc.

The inputs of dual PID Control are two actuating error signals $\xi_{1}$ and $\xi_{2}$, which can be generated by the two summing junctions which compare the desired and actual (feedback) angular velocities of the two wheel shafts of the robot. The outputs (Pulse Width Modulation) PWM 1 and PWM 2 are two control signals for two separately operating PMDC (Permanent Magnet DC) motors as show in Figure 5. Some intelligent methods for Position Control of DC Motor that can be added in this system are proposed in [39]. 
3. THE HARDWARE ARCHITECTURE OF THE TELE-ROBOTIC SYSTEM

The station of Remote control is composed of two principal parts:

- An application developed on a station connected to the robot called Application Server which is used as intermediary between the client and the robot, it reflects the received instructions of the server into understandable instructions by the robot.

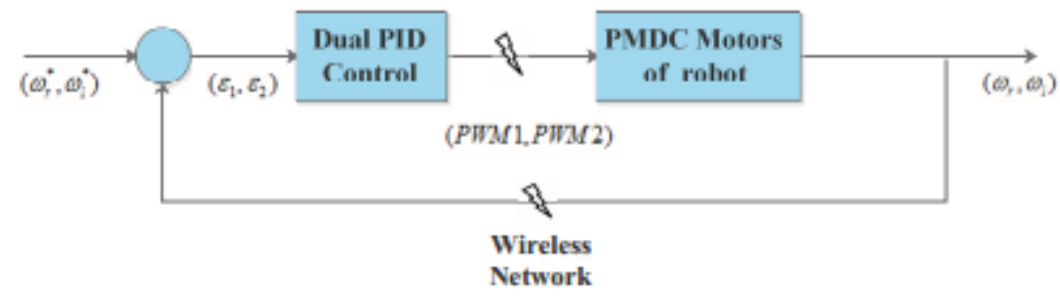

Figure 5. Control of DC motors

- Applets constituting the client using by the operator and will be the tool for the tele-operation of robot called Application Client. Three types of data flows are considered: High-level commands, visual feedback data and real-time motion control data.

The role of the Server is to receive the data coming from the client website and to transmit them to the robots to realize precise task using the Wireless Networking Protocol as see in Figure 6.

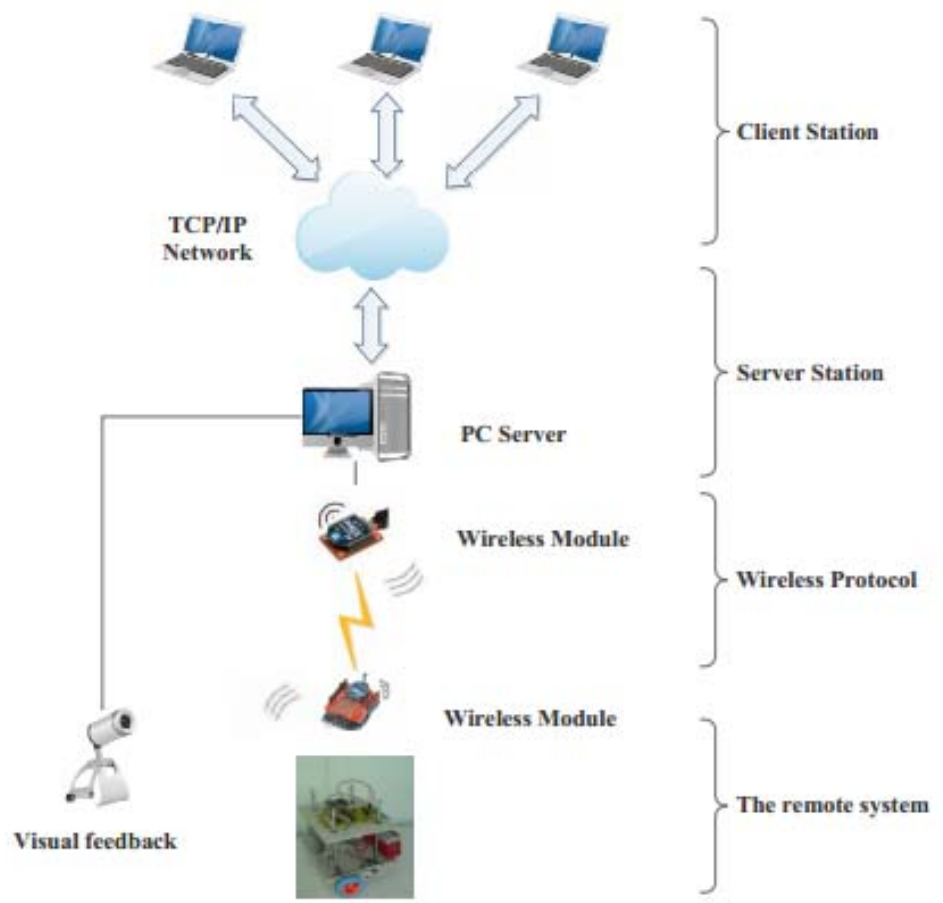

Figure 6. The Tele-robotic system

We use in this project a camera PixeLINK PL-B762F, The camera specifications are:

- $\quad$ Resolution: 752480.

- $\quad$ Sensor type: CMOS.

- $\quad$ FPS at full resolution: 60

- Interface: Firewire.

The experimental study was realized using a PC with the following material configuration 
- $\quad$ Intel Core 2 Quad @ 2.4GHz.

- 4GB RAM.

- $\quad$ Ethernet Network Device : RTL8101E Family PCI-E Fast Ethernet NIC.

- Wireless Network Device : Realtek RTL8187B Wireless 802.11g 54Mbps.

The system of the remote control allows a high degree of real-time interaction with a robotic system using VR environment to guarantee operability and safety. In this work, a design of two-wheeled mobile robot controlled by PC through wireless sensor network is suggested.

\subsection{Description of The Two-Wheeled Mobile Robot}

The developed a unicycle type mobile robot is produced with a form square as see in Figure 7 with two driving wheels on the same axis, and a wheel of insane the back. The weight is an important factor for our application because it plays the role of a resistive torque for the two motors.

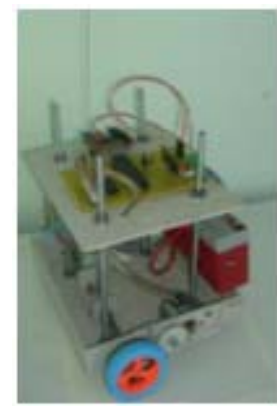

Figure 7. The Unicycle Mobile Robot

The robot is controlled by two DC motors, one for each wheel, where on board logic calculates reference wheel speeds and by the use of signals obtained from encoders. The frame having following dimensions in Table 1.

\section{Table 1. The dimensions of the mobile robot \\ Radius of the wheel $(\mathrm{cm}) 7.5$ \\ The distance between wheels $(\mathrm{cm}) 32$ \\ The total weight $(\mathrm{kg}) 1.650$}

The mobile robot specifications are:

- Two (02) DC Motors 251RPMw/Encoder 12V.

- 2A Dual Motor Controller.

- Arduino “Uno” (Rev 3).

- Arduino Wi-Fi Shield allows an Arduino board to connect to the Server using the 802.11 wireless specification (WiFi).

\section{RESULT AND ANALYSIS}

We present now, experimental results, the experimental system of tele-control has been presented; it is about a system of tele-operation whose design and implementation are based on the techniques of virtual reality and on the exploitation of technical communication networks. The remote control system allows a human operator to supervise and command the robot via the Internet networks and Wireless Networking Protocol. The station was developed at Laboratory of Power Systems, Solar Energy and Automation L.E.P.E.S.A, University of sciences and technology of Oran, Oran, Algeria.

The computer is connected to an Internet network via an Ethernet network interface. The robot is wirelessly connected to its server PC with Arduino Wi-Fi Shield module, these modules can communicate point to point, from one point to a PC, or in a mesh network. The role of Arduino Wi-Fi Shield is to transmit data to the systems using the Wireless network protocol. 


\subsection{Description of the Human/Robot Tele-Robotic Interface}

In order for robot tele-operation to perform well, the human-robot interface must be as efficient and as capable as possible. The interface provides tools to perceive the remote environment, to make decisions, and to generate commands. The operator is in a remote website with a machine connected to the Internet, The developed interface graphic provides the following features:

1. The control of robot.

2. The Animation of the Virtual robot.

3. The change of point view of the scene.

A 3D virtual model simulating the remote system robot allows viewing in real time the realization of its task (Figure 8). Figure 9 show the human/robot tele-robotic interface, the role of this interface is to enable the exchange of information

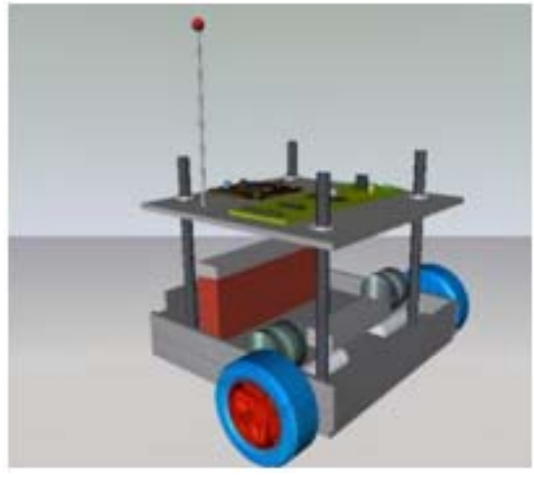

Figure 8. The 3D model of the robot

with the server and to follow the evolution of robots in the workspace. This interface provides all the necessary tools to ensure the control of the remote robot and to supervise the mobile robot with a console for the following the movement of the robot in its real and virtual workspace, these results show, as it might be expected, that it is more effective to control the robot by exploiting the control interface rather than controlling the robot manually.

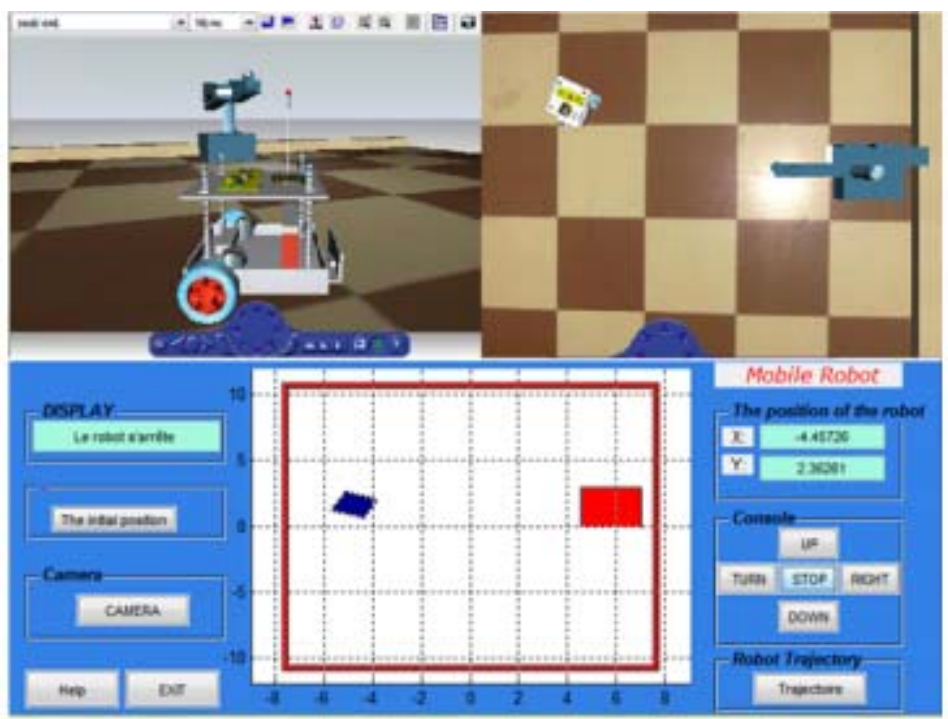

Figure 9. The 3D model of the robot 


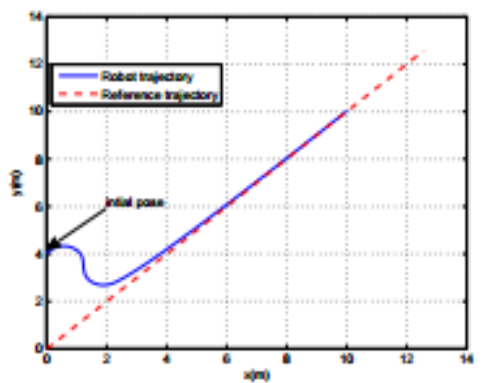

(a) Comparison between reference and real trajectory (line)

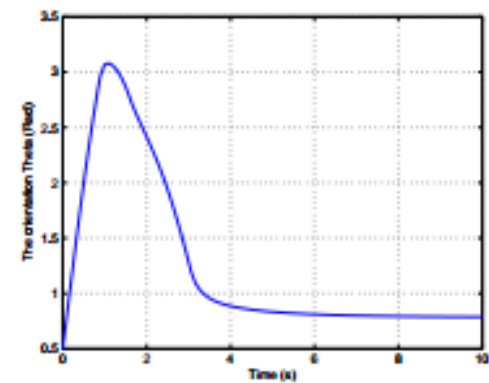

(b) The orientation $\theta$ of the robot

Figure 10. Following a line

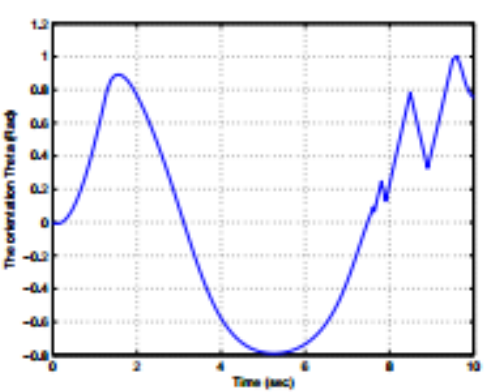

(b) The orientation $\theta$ of the robot reference and real trajectory

(Path)

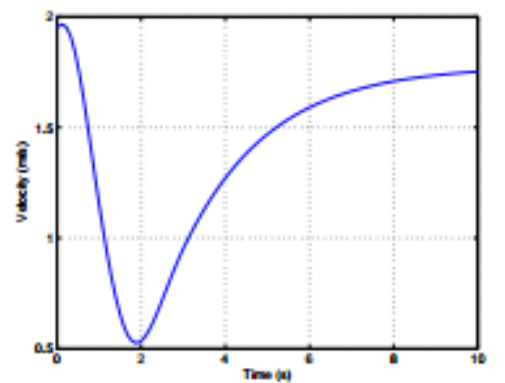

(c) The linear velocity $v$ of the robot

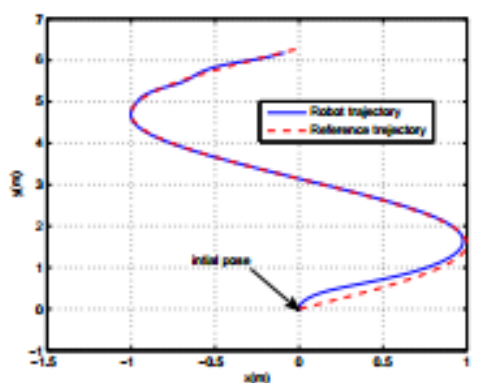

(a) Comparison between

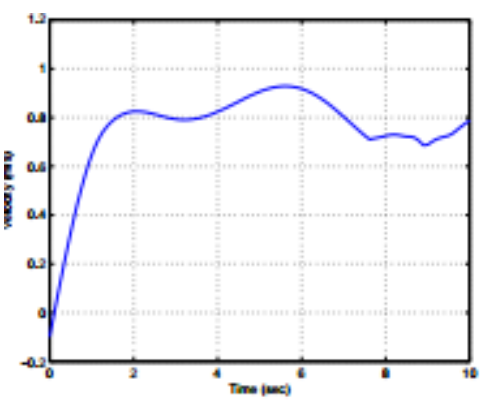

(c) The linear velocity $v$ of the robot

Figure 11. Following a Path

\subsection{Robot Trajectory}

We present now, experimental results and a detailed evaluation of different trajectories, and we compared the reference and real trajectories in the $\mathrm{x}-\mathrm{y}$ plane. The robot positions are measured via a camera PixeLINK PL-B762F whose frame rate is 60 images per second. The control is sent to the robot via wireless communication. In the Figure 10 (a), the task for a mobile robot is to follow a line on the plane (x,y). We carried out the experiment with the initial pose $P_{0}(x, y, \theta)=(0 ; 4 ; 28: 64)$, the robot starts at the origin but catches up to, and follows the moving goal. The orientation $\theta$ and the linear velocity $v$ of the robot are given in Figures 10 (b) and 10 (c), respectively. It can be observed that $\theta$ and $v$ are stabilized when the robot follows the reference trajectory.

Instead of a straight line we might wish to follow a path that is defined more generally as some locus on the x-y plane. The results are illustrated in Figures 11(a), 11(b) and 11(c). The orientation $\theta$ varies between $0.8 \mathrm{rad} / \mathrm{s}$ to $0.8 \mathrm{rad} / \mathrm{s}$. The linear velocity demand picks up smoothly and converges to a steady state value at the desired following distance, we notice that the translation of the two trajectories has roughly the same aspect except. The results proved the effectiveness of this system. Finally, this evaluation determines the most important performance criterion which is the accuracy.

\subsection{Discussion}

This work, a teleoperation system is developed using RV environment. The major issue of our application is ensuring the remote control of the mobile robot and integrating a path planning method in this system.

In Table 2, we compare different existing reasearch results, previous published papers on remote control used the VR application with a hybrid architecture of Web browser/server and client/server [26], these approachs are not tested with a path planning methods. Some authors were interested in simulation environment for testing of robotic algorithms without the use real implementation, simultaneous force and vision feedback to operate the robot after collision is added in [28], this research fails to present real implementation cases. 
A path planning method based on augmented reality (AR) interface with other manipulation system is pro posed in [30], the method is not tested in the remote control. Following these ideas, we tested a path planning method based on RV environment with real mobile robot, our system shows the real advantage of implementing this method for the remote control.

Table 2. Comparative study among different systems

\begin{tabular}{cccccc}
\hline Ref and Year & Augmented Reality & Virtual Reality & Path Planing & Real Implementation & Remote Control \\
\hline$[26](2013)$ & & $\bullet$ & & & $\bullet$ \\
{$[28](2014)$} & & $\bullet$ & & & \\
{$[29](2011)$} & & & $\bullet$ & & \\
{$[30](2014)$} & $\bullet$ & & $\bullet$ & $\bullet$ \\
\hline
\end{tabular}

\section{CONCLUSION}

In this paper, we presented an VR system intended to remote control of unicycle robot through an intuitive interface multimode human-machine. We tried to achieve this primary goal to provide a control system for the supervision and the following the evolution of real robot via the Internet and wireless network. The application requires the accuracy, for this purpose, we performed a comparative study of trajectories (reference and real) and we evaluated the performances of the system by using the forward, the inverse kinematics of the mobile robot and the 3D model. The analysis of results shows the performance of the system in term of accuracy, stability and convergence. The remote system offers several advantages, and allows an operator to oversee the execution of the tasks it performs. It also allows him to control the robot directly from their 3D model, thus facilitating the prediction of all sorts of operations that could affect the robot or the environment itself. It also offers the advantage of being able to run from any machine connected to the Internet.

In the near future, we intend to improve the system by using the augmented reality. This work can be applied to remote manipulation, remote manufacturing, entertainment, education and training. It is important to consider dynamic characteristics of mobile robot, which can be a reason of unexpected behavior of the robot when switching between control modes happens. For example, mobile robot cant be stopped at the same moment when switching happens due to inertia.

\section{REFERENCES}

[1] Sheridan. Thomas B. Telerobotics, automation, and human supervisory control. MIT Press Cambridge, USA, Reading, MA, 1992.

[2] EJP. Earon, et al., "Development of a multiagent robotic system with application to space exploration”, Advanced Intelligent Mechatronics. Proceedings. 2001 IEEE/ASME International Conference on, 2001, vol 2, pp. 1267-1272.

[3] LTC. Blitch, et al., "Semi-autonomous tactical robots for urban operations", in Proceedings of the 1998 IEEE international symposium on intelligent systems and semiotics (ISAS), 1998, pp. 783-794.

[4] IL. Ermolov, et al., "Internet control of mobile robots for pipe inspection/repair", in Proceedings of the 4th international workshop on computer science and information technologies, Sep 2002, pp. 18-20.

[5] T. Fong, C. Thorpe, "Vehicle teleoperation interfaces", Autonomous robots, vol. 11, no. 1, pp. 9-18, 2001.

[6] D. Carrier, W. David, "Soviet rover systems", in Proceedings of the AIAA Space Programs and Technologies Conference, Huntsville, AL, USA, 1992.

[7] D. Gage, "UGV history 101: A brief history of Unmanned Ground Vehicle (UGV) development efforts", Unmanned Systems Magazine, vol. 13, no. 3, pp. 9-16, 1995.

[8] W. Whittaker and L. Champeny, "Conception and development of two mobile teleoperated systems for TMI-2", in Proceedings of the International Meeting and Topical Meeting TMI-2 Accident,American Nuclear Society, 1988.

[9] D. Hainsworth, "Mine emergency survey vehicle: Numbat", in Proceedings of the 25th International Conference of Safety in Mines Research Institutes, Sep 1993, Pretoria, South Africa, pp. 7-18.

[10] T. Blackmon, L. Nguyen, C. Neveu, D. Rasmussen., et al., "Virtual reality mapping system for Chernobyl accident site assessment”, in Proceedings of SPIE Conference on Human Vision and Electronic Imaging IV, 1999, San Jose, CA, pp. 338-345.

[11] D. Hainsworth, “Teleoperation user interfaces for mining robotics”, Autonomous Robots, vol. 11, no 1, pp. 19-28, 2001.

[12] P. Ballou, “Improving pilot dexterity with a telepresent ROV”, in Proceedings of the IEEE Vehicle Teleoperations IJECE Vol. x, No. x, May 2015: 1 - 12 Interfaces Workshop - ICRA, 2001, San Francisco, CA.

[13] S. Rahnamaei and S. Sirouspour, “Automatic Viewpoint Planning in Teleoperation of a Mobile Robot”, Journal of Intelligent \& Robotic Systems, vol. 76, no 3-4, pp. 443-460, 2014.

[14] S. Jayaram, HI. Connache and KW. Lyons, "Virtual assembly using virtual reality techniques”, Computer-Aided Design, vol. 29, no.8, pp. 575-584, 1997. 
[15] KP. Beier, "Web-Based virtual reality in design and manufacturing applications", in Proceedings of First International Conference on Computer Application and IT in the Maritime Industries, 2001, Germany, pp 45-55.

[16] JY. Lee, H. Kim and K. Kim, "A web-enabled approach to feature-based modeling in a distributed and collaborative design environment”, Concurrent Engineering, vol. 9, no 1, pp. 74-87, 2001.

[17] N.Shyamsundar and R. Gadh, "Internet-based collaborative product design with assembly feature and virtual design spaces”, Computer Aided Design, vol. 33, no 9, pp. 637-651, 2001.

[18] H. Yamada, H. Kato and T. Muto, “Master-slave control for construction robot tele-operation”, Journal of Robotics and Mechatronics, vol. 15, no 1, pp. 5460, 2003.

[19] N. Tao., et al., "Vision-based virtual force guidance for tele-robotic system”, Computers \& Electrical Engineering, vol. 39, no 7, pp. 2135-2144, Oct 2013.

[20] D. Marini, R. Folgieri, D. Gadia, and A. Rizzi, "Virtual reality as a communication process”, Virtual Reality, vol. 16, no 3, pp. 233-241, 2012.

[21] Y.J. Xiong, S.Q. Li and M. Xie, "Predictive display and interaction of telerobots based on augmented reality", Robotica, vol. 24, no 4, pp. 447-453, 2006.

[22] B. Cooper, "Driving on the surface of Mars using the Rover Control Workstation", in In Proceedings of Space Ops, 1998, Tokyo, Japan.

[23] L. Nguyen, et al., "Virtual reality interfaces for visualization and control of remote vehicles", Autonomous Robots, vol. 11, no. 1 pp. 59-68, 2001.

[24] R. Zahariev and P. Petrova, “Approach with a virtual robot for control system design,” 2013.

[25] J. Aleotti and S. Caselli, "Physics-based virtual reality for task learning and intelligent disassembly planning", Virtual reality, vol. 15, no. 1, PP. 41-54, 2011.

[26] S. Liang, K. Chao and P. Ivey, "'VR-based wheeled mobile robot in application of remote real-time assembly", The International Journal of Advanced Manufacturing Technology, vol. 64, pp. 1765-1779, 2013.

[27] A. Vasalya and R. Agrawal, "Smart Telerobotic Surveillance System via Internet with Reduced Time Delay", International Journal of Robotics and Automation (IJRA), vol. 2, no 1, pp. 11-16, Mar 2013.

[28] KS. Niksirat, et al., "Teleoperation System with Force Feedback Joystick in Virtual Reality”, Artificial intelligence and Applications, vol. 1, no 2, 2014.

[29] B. Ouamri and Z. Ahmed-Foitih, "Adaptive neuro-fuzzy inference system based control of puma 600 robot manipulator”, International Journal of Electrical and Computer Engineering (IJECE), vol. 2, no 1, pp. 90-97, 2011.

[30] HC. Fang, SK. Ong and AYC. Nee, "A novel augmented reality-based interface for robot path planning”, International Journal on Interactive Design and Manufacturing (IJIDeM), vol. 8, no 1, pp. 33-42, 2014.

[31] R. Marn, et al., "A Multimodal Interface to Control a Robot Arm via the Web: A Case Study on Remote Programming”, IEEE transactions on industrial electronics, vol. 52, no 6, pp. 1506-1520, Dec 2005.

[32] W. Lihui, et al., "Remote realtime CNC machining for web-based manufacturing", robotic computer-integrated manufacturing, vol. 20, no 6, pp. 563-571, 2004.

[33] VR. Kamat, RR. Lipman , "Evaluation of standard product models for supporting automated erection of structural steelwork”, Automation in Construction, vol. 16, no 2, pp. 232-241, 2007.

[34] SK. Ong, L.Jiang, AYC. Nee, “An internet-based virtual CNC milling system”, Journal of advanced manufacturing Technology, vol. 20, no 1, pp. 20-30, 2002.

[35] C. Peter. Robotics, vision and control: fundamental algorithms in MATLAB, Vol. 73. Springer Science \& Business Media, 2011

[36] J. Rubio, et al., “Inverse kinematics of a mobile robot”, Neural Computing and Applications, vol. 23, no 1, pp. 187194, 2013.

[37] Dorf R. Bishop R. Modern Control Systems, 8th edition. Addison-Wesley, Reading, California, 1998.

[38] Ogata K. Modern Control Systems, 4th edition. Prentice-Hall of India, Reading, New Delhi, 2002.

[39] NM. Yazdani, AY. Seqerloo, "Performance Comparison between Classic and Intelligent Methods for Position Control of DC Motor”, International Journal of Electrical and Computer Engineering (IJECE), vol. 4, no 3, pp. 366-371, 2014.

\section{BIOGRAPHIES OF AUTHORS}

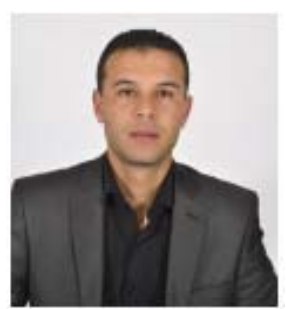

Ibari Benaoumeur was born in Tighennif-Mascara, Algeria in mars 1983. Has received the Engineering Degree in Automatic in 2007, the Magister in Automatic from University of Sciences and Technology of Oran USTO (ORAN - Algeria) in 2010, and now he is Phd student in the same University. Since 2011, he is lecturer and researcher member of the Electronics Department, University of Chlef Algeria. His current research interests include Robotics, Computer automation, control of industrial processes and supervision, Identification of process, soft computing. 

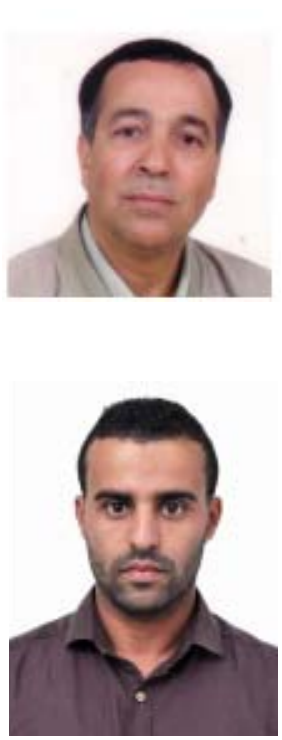

Ahmed-Foitih Zoubir has received the Engineering Degree in Electronics in 1980, the Magister in Automatic in 1988, and the PhD degree in 2004 from the University of Sciences and Technology of Oran USTO (ORAN, Algeria). Since 1982, he is lecturer and researcher member of the Electronics Department, U.S.T.O. Since 2012, he is Professor at the USTO Oran and head of Augmented Reality team which is implied in several French and European projects. He is currently a full professor with the University of Sciences and Technology of Oran. His current research interests include Robotics, Computer automation, control of industrial processes and supervision, Identification of process, soft computing.

Hanifi Elhachimi Amar Reda was born in Oran, Algeria on January 1987. He received the License and Master Degree in Industial Data processing in 2009 and 2011 respectivly from University of Sciences and Technology of Oran USTO (ORAN - Algeria) and now he is Phd student in the same university. His current research interests include Robotics, automation control, control of industrial processes and supervision. 